\title{
Complications associated with different insertion techniques for Hickman catheters
}

\author{
Zahoor Ahmed, Zahoor Mohyuddin
}

\begin{abstract}
Summary
A dependable central venous access is an essential prerequisite for the delivery of care to the cancer patients. This has become easier since the introduction of indwelling central venous catheters. However, pancytopenia, often encountered in haematological malignancies, does influence the morbidity associated with these catheters. In this study the effects of two different placement techniques of Hickman catheters were evaluated. A total of 177 Hickman catheters were inserted in patients suffering from haematological malignancies. In 112 patients the catheters were placed percutaneously into subclavian veins without prior tunnelling; in the other 65 patients these were introduced, after having been tunnelled, into cephalicl external jugular veins by a cut-down technique. The catheters remained in use in each patient for between 18 and 253 days. Excessive bleeding and haematoma formation occurred in a significantly higher proportion of patients from the cut-down group (61\% and $41 \%$, respectively) compared to patients from the percutaneous group ( $8 \%$ and $0 \%$, respectively). These in turn had a profound impact on the incidence of infective complications. Catheter exit site infection, tunnel infection and septicaemia were observed in $26 \%, 18 \%$, and $41 \%$, respectively, of the patients from the cut-down group, while in patients from the percutaneous group, skin exit site infection was noted only in $7 \%$ and septicaemia in $19 \%$ of patients. It was concluded that the placement technique of these catheters exerts a significant influence on the immediate as well as delayed complications. The results indicate that the insertion technique requiring minimal dissection is more beneficial. It is therefore recommended that percutaneous insertion without tunnelling should be the technique of choice for catheter placement.
\end{abstract}

Keywords: Hickman catheter; chemotherapy; placement techniques

Acquisition of a reliable and sustained central venous access is a crucial factor in the provision of comprehensive care to the cancer patients. The adverse effects of antineoplastic drugs in the form of phlebitis, thrombosis, and fibrosis, impose limitations on the repeated use of peripheral veins for this purpose. An adequate central venous access therefore, by obviating the dependency on fragile peripheral veins, contributes significantly towards the supportive care needed to sustain these patients on a long-term basis.

Since their introduction, indwelling Hickman catheters have proved to be an ideal means for the delivery of chemotherapeutic agents and other supportive care required for the recovery of these moribund patients. However, the technique of their placement may influence the associated morbidity and affect the outcome. In this retrospective study, two different techniques of placement of Hickman catheters were adopted. In one group the catheters were inserted using a cut-down method while in the second group a percutaneous technique was used. This study compares the effects of placement technique on outcome.

\section{Patients and methods}

Written informed consent was obtained from all patients. A total of 177 Hickman catheters were inserted. In 65 patients the catheters were inserted using the cut-down method into either the external jugular vein ( 58 patients) or the cephalic vein (seven patients) in the deltopectoral groove. In 112 patients the catheters were positioned through a peel-away sheath using a percutaneous technique. The youngest patient was a four-year-old child and the eldest was 80 years old but the majority were between 25 and 43 years old; 118 were male and 59 female. In the cut-down group, the catheters were first tunnelled through the anterior chest wall before being negotiated into the cephalic or external jugular veins. In the percutaneous group the catheters were inserted directly into subclavian veins through a peel-away sheath without prior tunnelling. The catheters used were Hickman dual-lumen vascular access catheters. All the catheters were placed under local anaesthesia using $2 \%$ plain xylocaine infiltration except in the four-year-old child who was given a general anaesthetic. The length of each catheter to be inserted was determined by placing it presternally along the presumed course of the subclavian vein and superior vena cava, so that, with the dacron felt cuff positioned just beneath the skin at the exit site, the tip of the catheter lay at the superior vena cava / right atrial junction. The patency of all the catheters was ensured by flushing them with heparinised saline after each use or at least once a day. 
Table Complications in two groups of patients with Hickman catheters (percentages in parentheses)

\begin{tabular}{lcc}
\hline Complications & $\begin{array}{l}\text { Cut-down technique } \\
(n=65)\end{array}$ & $\begin{array}{l}\text { Percutaneous technique } \\
(n=112)\end{array}$ \\
\hline Immediate & $40(61)$ & $9(8)$ \\
Excessive bleeding & $27(41)$ & $0(0)$ \\
Haematoma formation & $3(4)$ & $6(5)$ \\
Malposition & $0(0)$ & $6^{\star}(5)$ \\
Pneumothorax & & \\
Delayed & $6(9)$ & $8(7)$ \\
Catheter thrombosis & $0(0)$ & $3(3)$ \\
Spontaneous catheter expulsion & $4(6)$ & $3(3)$ \\
Catheter rupture & $17(26)$ & $8(7)$ \\
Exit site infection & $12(18)$ & $0(0)$ \\
Tunnel infection & $27(41)$ & $21(21)$ \\
Septicaemia & & \\
\hline
\end{tabular}

* Bilateral in one. taneous groups, respectively. These patients had multiple episodes of septicaemia which were effectively treated with appropriate antibiotics in all except 19 patients who continued to grow various organisms from blood cultures despite intensive therapy.

Even when the comparative analysis of the incidence of infective complications was made after exclusion of the patients in whom the catheters were removed prematurely, the cutdown group was found to be at a significant disadvantage. The rates of exit site infection, tunnel infection, and septicaemia are shown in the table.

The reasons for removal of the catheters were septicaemia in 19 patients, catheter blockage in 14, catheter breakage in seven, death in 27 ( 11 from the cut-down group and 16 from percutaneous group), and completion of treatment in 110 patients. Even in the presence of septicaemia, catheters were not removed from patients in whom there were no external signs of infection (in the form of redness at the insertion site), because septicaemia in these patients was not attributed to the catheters. Neutropenic patients have many other sources of infection and removal of catheters in these patients would rather jeopardise management, as it would result in the loss of an effective route for the administration of antibiotics.

Avoidance of surgical dissection and tunnelling significantly reduced the number of immediate complications and the incidence of infective complications.

\section{Discussion}

The care of a cancer patient presents a complex and agonising dilemma, both for the patient and for the managing team. Apart from the other multiple requirements, the availability of a reliable long-term central venous access is of prime importance for the administration of chemotherapeutic agents and other supportive care needed for the recovery of these terminally ill patients. Since their inception, Hickman catheters have proved their efficacy in achieving this objective. Insertion of these catheters has more often been achieved using a cut-down technique into the cephalic vein or the external or internal jugular veins. ${ }^{4}$ Although these are reliable sites of access, there is a high incidence of morbidity. In particular, attempts at cannulation of the cephalic veins in the deltopectoral groove are often hampered by the inadequate size of these veins. We have ourselves encountered difficulties in cannulating cephalic veins due to inadequacy of their size and abandoned this route after utilising it for only seven patients. The technique of percutaneous insertion of Hickman catheters into subclavian veins, however, was found to be expeditious and beneficial. ${ }^{5}$ The foremost advantage is that it obviates the need for an operating room, a requirement for any surgical procedure such as an exposure of a vein by cut-down. Catheter placement by the percutaneous route, on the other hand, can be accomplished in any sterile surroundings, paying adequate attention to asepsis.
Septicaemia was documented in $41 \%$ and $19 \%$ of patients from the cut-down and percu- 


\section{Hickman catheter characteristics}

- Hickman catheters provide reliable long-term venous access

- placement technique influences morbidity

- cut-down method enhances haematoma formation, tunnel infection and septicaemia

- need for operating room facility for cut-down method increases costs significantly

- percutaneous insertion is easy, quicker, and cost-effective

- fluoroscopic monitoring not essential for correct positioning

Box 1

Various modalities have been employed to ascertain that the catheter tip is located in the desired position. Intra-operative monitoring by roentgenographic, fluoroscopic or electrocardiographic control ${ }^{6-8}$ certainly facilitates the procedure but their availability is not mandatory. The catheter, advanced into a major capacious vein, slides into the superior vena cava without encountering much resistance. Their position can easily be verified by a simple chest $\mathrm{X}$-ray and any doubts concerning the catheter tip location can be resolved by prompt venography through the catheter. In our study, the malplacement of the catheter tip occurred in three patients from the cut-down group compared with six patients from the percutaneous group. In one patient from the cut down group, the catheter tip, inserted through the external jugular vein, was deflected into anterior jugular vein because of the anomalous venous communication while in the other two patients it became lodged in the inferior vena cava. In the percutaneous group, the catheter tips were deflected into left innominate veins. In all cases, the catheters were repositioned correctly.

Patients suffering from haematological malignancies usually have a low platelet count and thus are prone to excessive bleeding from wounds inflicted during surgery. To impede the descent of infection, the principle of maintaining an adequate distance between the catheter exit site and the site of entry into the recipient vein holds good, but in view of the compromised homeostasis in thrombocytopenic patients any attempt to create a subcutaneous tunnel, no matter how efficiently done, does result in haematoma formation. This complication could be a crucial factor in triggering the onset of an infective process in patients with particularly low resistance due to neutropenia. The percutaneous insertion technique is cleaner, quicker (taking about 20 minutes), and a much less traumatic procedure. Extremely little surgical interference, lack of any surgical wounds and haematoma formation keep the infective process at bay. Broadwater $e t$ $a l^{9}$ have reported their experience of the percutaneous insertion of catheters into subclavian veins in a very large population of patients with the achievement of significant success at a minimal cost. Similarly Huijgens et $a l^{10}{ }^{10}$ encouraged by the low associated morbidity, have strongly advocated adoption of the percutane- ous technique for the insertion of Hickman catheters. In addition to the placement technique, an important consideration influencing the longevity of the catheter life-span is patient education, which may help to reduce the frequency of complications and minimise the associated morbidity.

\section{Conclusion}

Apart from the other factors, the route of placement of central venous catheters has a significant effect on morbidity. The findings from this study suggest that, because of the enhanced propensity of bleeding attendant upon compromised haemostasis, patients needing intensive therapy for haematological malignancies are best managed by central venous catheters that require minimum dissection for insertion. Percutaneous insertion without tunnelling is a cost-effective, convenient and reliable method of achieving prolonged venous access for protracted chemotherapy and supportive care with minimum accompanying morbidity. Therefore, it is recommended that, while maintaining meticulous sterility, percutaneous insertion without tunnelling should be the technique of choice for the procurement of secure central venous access in these moribund patients.

\section{Maintenance of Hickman catheter}

- for patency, flush each lumen of Hickman catheters with $10 \mathrm{ml}$ of normal saline followed by $2-3 \mathrm{ml}$ of heparinised saline $(100 \mathrm{U} / \mathrm{ml})$ after each use, or at least once a day with heparinised saline only when not used

- catheter exit site to be cleaned with antiseptic solution and dressed with sterile dressing which should be changed every 72 hours or when soiled

- catheter hubs to be kept well covered with sterile gauze

- declotting of thrombosed catheter to be attempted with $1 \mathrm{ml}$ of urokinase solution (5000 $\mathrm{U} / \mathrm{ml}$ )

- catheter removal not helpful in treatment of septicaemia in the absence of exit site or tunnel infection

Box 2

\section{Patient education}

- clear explanation and demonstration to the patients/relatives about catheter care

- demonstrate how to flush the catheter with heparin saline

- demonstrate how to clean the skin and change the dressing

- stress need to avoid accumulation of sweat in catheter region

- advise to seek immediate help in case of difficulty in flushing the catheter, suspicion of catheter migration, any discharge from the exit site, pain/redness over the catheter tunnel, and any fever 
1 Pessa ME, Howard RJ. Complications of Hickman catheters. Surg Gynecol Obstet 1985;161:257-60.

2 Jansen RFM, Wiggers T, van Geel BN, et al. Assessment of Jansen RFM, Wiggers T, van Geel BN, et al. Assessment of
insertion techniques and complication rates of dual lumen insertion techniques and complication rates of dual lumen central venous catheters in patients with
malignancies. World $\mathcal{F}$ Surg 1990;14:101-6.

3 Mofti AB, Haque MM. Central venous access for cance chemotherapy: an easy technique. Emirates Med $\mathcal{F} 1987 ; 5$ $167-9$.

4 Heimbach DM, Ivey TD. Technique for placement of a permanent home hyperalimentation catheter. Surg Gynecol Obstet 1976;143:635-6.

5 Dimitrios AL, Mucha Jr P. A simplified technique for the placement of permanent central venous catheters. Sur Gynecol Obstet 1982;154:248-50.
6 Redo SF, Dinner MH. Placement of central venous catheters by cut down with electrocardiogram. Surg Gynecatheters by cut down with
col Obstet 1993;177:49-53.

7 Luks FI, Picard DL, Pizzi WF. Electrocardiographic guidance for percutaneous placement of central venous catheters. Surg Gynecol Obstet 1989;169:157-8.

8 Wilson RG, Gaer TA. Right atrial electrocardiography in placement of central venous catheters. Lancet 1988;1:462-

9 Broadwater JR, Henderson MA, Bell JL, et al. Outpatien percutaneous central venous access in cancer patients. $A m$ f Surg 1990;160:676-80.

10 Huijgens PC, Strack van Schijnder R, Kampman I, et al. A prospective study of untunneled subclavian vein catheters in hematoncology patients. Neth $\mathcal{F}$ Med 1991;38:153-8.

\section{Medical Anniversary \\ RENÉ LAENNEC, 17 February 1781}

René Théophile Hyacinthe Laennec (1781-1826) was born at Quimper, Finistère, France, son of a barrister and nephew of the medical Rector of the University of Nantes. He studied medicine in Nantes for five years throughout the Revolution and thereafter in Paris with Corvisart and Dupuytren. He held appointments at the Necker and La Charite hospitals. In 1822 he attained professorial status, produced his monumental book on breath sounds, was elected a member of the Academy of Medicine (1823), and in 1824 he was married and awarded the Legion d'Honneur. One of his early stethoscopes was a cardboard tube but later he selected a wooden cylinder a quarter of an inch in diameter and about 12 inches long. In his book de l'Auscultation Mediate (Sir William Hale-White, published by Bale, Sons and Danielsson 1923) he describes both normal and diseased lungs, mostly due to tuberculosis, from which he died far too young. $-D G$ fames 